\title{
Detection of the ellipsoidal and the relativistic beaming effects in the CoRoT-3 lightcurve
}

\author{
T. Mazeh and S. Faigler \\ School of Physics and Astronomy, Raymond and Beverly Sackler Faculty of Exact Sciences, Tel Aviv University, \\ Tel Aviv 69978, Israel \\ e-mail: mazeh@post.tau.ac.il \\ Received 9 August 2010 / Accepted 21 September 2010
}

ABSTRACT

\begin{abstract}
CoRoT-3b is a 22 Jupiter-mass massive-planet/brown-dwarf object, orbiting an F3-star with a period of 4.3 days. We analyzed the out-of-transit CoRoT-3 red-channel lightcurve obtained by the CoRoT mission and detected the ellipsoidal modulation, with half the orbital period and an amplitude of $59 \pm 9 \mathrm{ppm}$ (parts per million), and the relativistic beaming effect, with the orbital period and an amplitude of $27 \pm 9 \mathrm{ppm}$. Phases and amplitudes of both modulations are consistent with our theoretical approximation.
\end{abstract}

Key words. methods: data analysis - planetary systems - stars: individual: CoRoT-3

\section{Introduction}

Close binary stellar systems display two well-known periodic photometric modulations - the ellipsoidal effect, due to the distortion of each component by the gravity of its companion (see a review by Mazeh 2008), and the reflection/heating effect (referred to here as the reflection effect), induced by the luminosity of each star, which affects only the close side of its companion (e.g., For et al. 2010). These two effects can be observed even for non-eclipsing binaries, but are much easier to study in eclipsing binaries, where the binarity of the system and the phases of the orbital motion are well known from the observations of the eclipses. Most algorithms that analyze lightcurves of eclipsing binaries, such as EBOP (Etzel 1980; Popper \& Etzel 1981) and its derivative EBAS (Tamuz et al. 2008), WD (Wilson \& Devinney 1971), and ELC (Orosz \& Hauschildt 2000), include by default these two effects in their model of the out-of-eclipse lightcurve.

A much smaller and less studied photometric modulation is the relativistic beaming effect, sometimes also called Doppler boosting, induced by the stellar motion relative to the observer $V_{\text {rel }}$, whose amplitude is on the order of $V_{\text {rel }} / c$, where $c$ is the velocity of light. Before the era of space photometry this effect has been noticed only once, by Maxted et al. (2000), who observed KPD $1930+2752$, a binary with a very short period, of little longer than $2 \mathrm{~h}$, and a radial-velocity amplitude of $350 \mathrm{~km} \mathrm{~s}^{-1}$. The beaming effect of that system, which should be on the order of $10^{-3}$, was hardly seen in the photometric data.

Space photometry, which was developed to detect the minute transits of exoplanets, has substantially improved the precision of the produced lightcurves. The CoRoT (Rouan et al. 1998; Baglin et al. 2006; Auvergne et al. 2009) and Kepler (Borucki et al. 2010; Koch et al. 2010) missions are producing hundreds of thousands of continuous photometric lightcurves with timespan of tens and hundreds of days, with precision that can reach as high as $10^{-3}-10^{-4}$ per measurement. It was therefore anticipated that CoRoT and Kepler should detect all three effects (e.g., Drake 2003), in particular the beaming effect for both planets (Loeb \& Gaudi 2003) and eclipsing binaries (Zucker et al. 2007).
As predicted, van Kerkwijk et al. (2010) detected in the Kepler lightcurve the ellipsoidal and the beaming effect of two eclipsing binaries, KOI 74 and KOI 81 (Rowe 2010). They used the radial-velocity photometric beaming effect to derive the mass of the secondary in the two systems and showed that in both cases it was probably a white dwarf. Welsh et al. (2010) identified the ellipsoidal effect in the Kepler data of HAT-P-7, a system with a known planet of 1.8 Jupiter masses $\left(=M_{\text {Jup }}\right)$ and a period of 2.2 days (Pál et al. 2008). Snellen et al. (2009) detected in the CoRoT data the reflection effect of CoRoT-1.

In this paper, we report the detection of the ellipsoidal and the beaming effects of CoRoT-3, induced by its massiveplanet/brown-dwarf companion. CoRoT-3b (Deleuil et al. 2008) is a 22 Jupiter-mass object, orbiting an F3-star with a period of 4.26 days. The stellar rotation is probably synchronized with the orbital period. We analyzed the CoRoT-3 out-of-transit redchannel lightcurve and detected two modulations, one with the orbital period and the other with its first harmonic. We attributed the two modulations to the beaming and the ellipsoidal effects, respectively, as their phases and amplitudes were consistent with our order-of-magnitude approximation. Section 2 presents our data analysis, Sect. 3 compares our findings with theoretical approximations, and Sect. 4 summarizes our results.

\section{Data analysis}

CoRoT-3 was discovered (Deleuil et al. 2008) in the data obtained during the first long run of the CoRoT mission - LRc01, which lasted for $152.012 \mathrm{~d}$, from May 26 until October 25, 2007 (for details about this run see Cabrera et al. 2009). The optics of the mission include a bi-prism that disperses the stellar light into three channels, red, green, and blue, the sum of which is called the white channel. For bright stars, including CoRoT-3, the light intensity coming through each of the three channels is available. We used the so-called N2 data level (Baudin et al. 2006) of CoRoT-3, which is now public.

As the modulations we searched for were quite small, we had to prepare and clean the data before searching for any periodic effect. We decided to concentrate on the red-channel data, because this channel included most of the stellar light detected 
by CoRoT, and the other two channels only added noise to the data (see below). Snellen et al. (2009) adopted a similar strategy when analyzing the lightcurve of CoRoT-1. This section describes how we "cleaned" the data, removed the long-term variation, and searched for the periodic modulation with the orbital period and its harmonics.

\subsection{Cleaning the lightcurve}

The "cleaning" of the CoRoT-3 lightcurve had the following stages:

- rebinning: Corot-3b was detected before the CoRoT run was completed and therefore the cadence of the observations was changed during the run - the first part of the lightcurve is composed of $512 \mathrm{~s}$ exposures, while the later part contains $32 \mathrm{~s}$ exposures. Since we were interested in modulations with periods equal to or longer than half the orbital period, at about $2.1 \mathrm{~d}$, the entire light curve was re-binned into $512 \mathrm{~s}$ bins. Altogether, we derived 22072 valid measurements;

- removing transits: 1165 measurements taken during the transits of CoRoT-3 were removed from the analysis;

- jump removal: one "jump", at CoRoT HJD of 2746.99, probably caused by a "hot pixel" event, was identified and corrected. The counts after the jump were adopted to the stellar flux before the jump, while 24 measurements following the jump were removed;

- outlier removal: we identified 106 outliers by calculating the running median and RMS around each point, and rejecting measurements that differed by $4 \sigma$ or more from their corresponding median. We were left with 20801 data points.

\subsection{Long-term detrending with a cosine filter}

The CoRoT-3 lightcurve clearly contained a long-term variation, as can be seen in Fig. 1, where we plot the relative redchannel flux, after subtracting and dividing the original flux by its median.

To remove this trend we used a discrete cosine transform (Ahmed et al. 1974), adopted to the unevenly spaced data we had in hand. We fitted the data with a linear combination of the first $N$ low-frequency cosine functions

$$
\left\{f_{i}\left(t_{j}\right)=\cos \left(\frac{2 \pi}{2 T} i \times t_{j}\right) ; i=0, N\right\} \text {, where } N=\operatorname{Round}\left(\frac{2 T}{4 P_{\text {orb }}}\right)=18,(1)
$$

$T=152.012 d$ is the timespan of the observations, $P_{\text {orb }}=$ $4.2568 d$ is the orbital period of CoRoT-3, and $t_{j}$ is the timing of the $j$ th measurement. The fitting finds the linear coefficient $a_{i}$ for each of the cosine functions, so that the fitted model is

$\mathcal{M}\left(t_{j}\right)=\sum a_{i} f_{i}\left(t_{j}\right)$

We then subtracted the model $\mathcal{M}\left(t_{j}\right)$ from the lightcurve.

The general idea was to perform a high-pass filter, so we removed all the low-frequency cosine components of the lightcurve without altering the periodic modulation of the orbital period. In a similar manner we also removed the satellite and earth frequencies, which appeared in the N2 data (e.g., Mazeh et al. 2009).

Figure 1 shows the red-channel lightcurve before and after the removal of the long-term trend and the satellite and earth modulation. The RMS of the cleaned lightcurve is $904 \mathrm{ppm}$ (parts per million). A similar analysis of the blue- and greenchannel data yielded lightcurves with highly correlated noise, of an RMS of 1600 and 2000 ppm, respectively. The white-channel

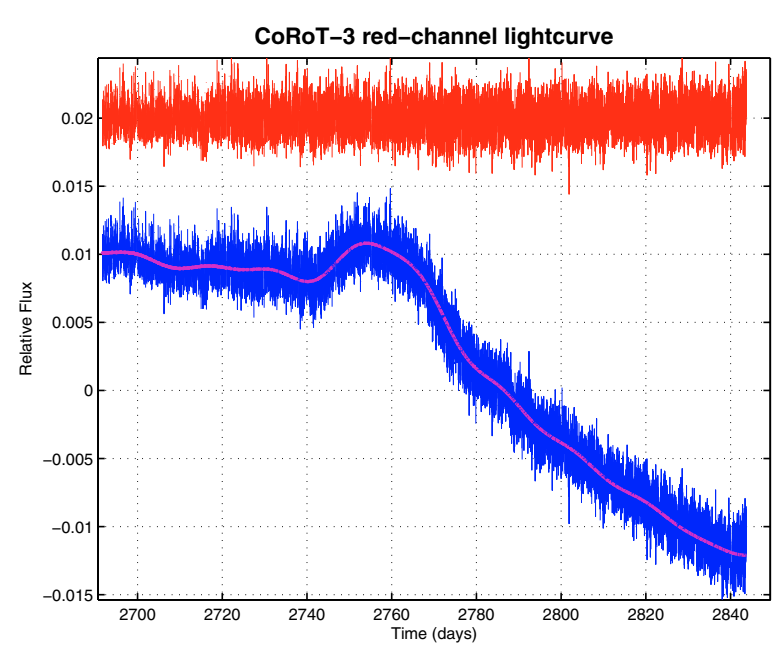

Fig. 1. The lightcurve of CoRoT-3, before (blue) and after (red) detrending. The long-term model is presented by the wide line. Time is the CoRoT time $=\mathrm{BJD}-2451545.0$. Flux is relative to the mean redchannel flux. The detrended lightcurve is shifted by 0.02 .

lightcurve, which includes the blue and the green data, is affected by similar problems. These results supported our decision to consider the red-channel data only.

\subsection{Fitting the amplitudes of the ellipsoidal, beaming, and reflection effects}

We finally proceeded to fit a model that includes the ellipsoidal, beaming and reflection effects. We approximated each of the three effects using pure sine/cosine functions, relative to the middle of the transit, $t_{\text {tran }}$, denoted as phase zero. The reflection and the beaming effects were approximated by sine and cosine functions, respectively, with the orbital period, and the ellipsoidal effect by a cosine function with half the orbital period (see next section). In this approximation, we expressed the stellar flux modulation $\Delta F$ as a fraction of the averaged flux $\bar{F}$, and a function of $\hat{t} \equiv t-t_{\text {tran }}$ :

$$
\begin{aligned}
& \frac{\Delta F_{\text {ellip }}(\hat{t})}{\bar{F}}=-A_{\text {ellip }} \cos \left(\frac{2 \pi}{P_{\text {orb }} / 2} \hat{t}\right), \\
& \frac{\Delta F_{\text {beam }}(\hat{t})}{\bar{F}}=A_{\text {beam }} \sin \left(\frac{2 \pi}{P_{\text {orb }}} \hat{t}\right), \\
& \frac{\Delta F_{\text {refl }}(\hat{t})}{\bar{F}}=-A_{\text {refl }} \cos \left(\frac{2 \pi}{P_{\text {orb }}} \hat{t}\right),
\end{aligned}
$$

where the coefficients, $A_{\text {ellip }}, A_{\text {beaming }}$, and $A_{\text {refl }}$ are all positive.

We therefore fitted the cleaned, detrended lightcurve of CoRoT-3 with a 5-parameter model, $\mathcal{M}_{\mathrm{ebr}}$, consisting of two frequencies

$$
\begin{aligned}
\mathcal{M}_{\mathrm{ebr}}\left(t_{j}\right)= & a_{0}+a_{1 \mathrm{c}} \cos \left(\frac{2 \pi}{P_{\mathrm{orb}}} \hat{t}_{j}\right)+a_{1 \mathrm{~s}} \sin \left(\frac{2 \pi}{P_{\mathrm{orb}}} \hat{t}_{j}\right) \\
& +a_{2 \mathrm{c}} \cos \left(\frac{2 \pi}{P_{\mathrm{orb}} / 2} \hat{t}_{j}\right)+a_{2 \mathrm{~s}} \sin \left(\frac{2 \pi}{P_{\mathrm{orb}} / 2} \hat{t}_{j}\right)
\end{aligned}
$$

as performed by Sirko \& Paczyński (2003). The fitting process could find any value, positive or negative, for the five parameters. However, we did expect $a_{1 \mathrm{~s}}$ to represent the beaming effect and therefore be positive, $a_{1 \mathrm{c}}$ to represent the reflection effect and therefore be negative, $a_{2 \mathrm{c}}$ to represent the ellipsoidal 
Table 1. The fitted coefficients and the theoretical expected amplitudes of the three effects of CoRoT-3.

\begin{tabular}{lrcl}
\hline \hline Coefficient & $\begin{array}{c}\text { Derived value } \\
(\mathrm{ppm})\end{array}$ & $\begin{array}{c}\text { Expected amplitude } \\
(\mathrm{ppm})\end{array}$ & Effect \\
\hline$a_{1 \mathrm{c}}$ & $-14 \pm 9$ & $-\alpha_{\text {refl }}(7.2 \pm 0.3)$ & Reflection \\
$a_{1 \mathrm{~s}}$ & $27 \pm 9$ & $\alpha_{\text {beam }}(29 \pm 0.5)$ & Beaming \\
$a_{2 \mathrm{c}}$ & $-59 \pm 9$ & $-\alpha_{\text {ellip }}(32 \pm 5)$ & Ellipsoidal \\
$a_{2 \mathrm{~s}}$ & $0.1 \pm 9$ & - & - \\
\hline
\end{tabular}

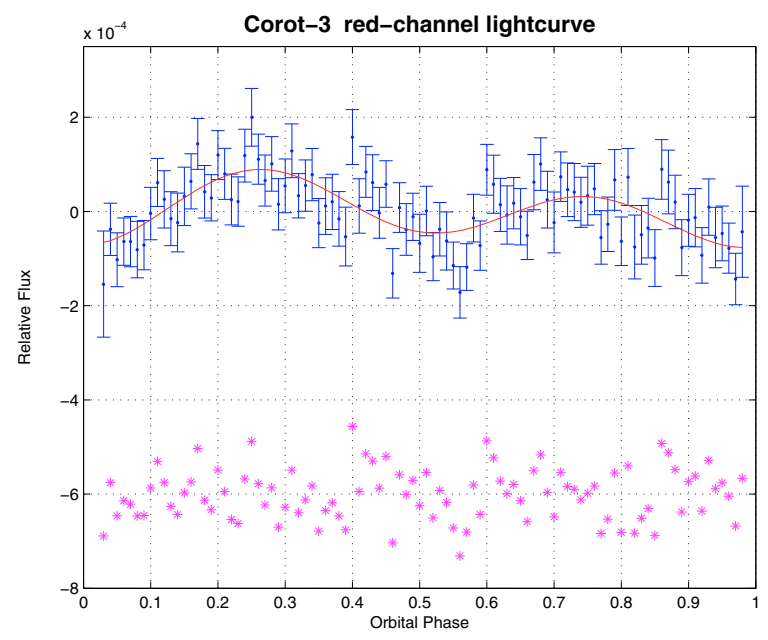

Fig. 2. The folded cleaned lightcurve of CoRoT-3, binned into 100 bins, with the fitted model. The residuals are plotted at the bottom of the figure.

effect and therefore be negative, and $a_{2 s}$ to be close to zero. The last expectation is important because it ensures that the ellipsoidal modulation is detected with the correct phase. The $a_{0}$ parameter was induced to remove any DC component left in the data. Obviously, the expected absolute values of the three coefficients depended on the parameters of the CoRoT-3 system. Thus, after the analysis was performed we were able to verify our results by comparing them with the expected values.

The results of the fitting are given in Table 1 (with some order-of-magnitude theoretical expectations; see next section) and plotted in Fig. 2. In the figure one can easily discern the ellipsoidal modulation, with half the orbital period, and the beaming effect, which causes the difference between the two peaks. One indication of the consistency of our results with the expected modulations is the correct sign of the three first coefficients and that the fourth coefficient is smaller than the third one by at least one order of magnitude. This suggests a close agreement between the orbital phase of the ellipsoidal modulation and that of the transit. We note that the errors in the four coefficients are all $9 \mathrm{ppm}$, or somewhat smaller than $10^{-5}$ in relative flux. This precision does not allow a significant detection of the reflection modulation. However, the detection of both the ellipsoidal and the beaming effect is highly significant. A bootstrap test indicates that the probability of detecting the beaming modulation by chance is $2 \times 10^{-4}$.

\section{Theoretical approximation}

This section presents our theoretical approximations of the ellipsoidal, beaming, and the reflection effects. We are not interested in detailed calculations, which depend on specific models. For example, to calculate the ellipsoidal modulation one could integrate the light originating from the individual surface elements of the rotating star, which is slightly deformed by the tidal force induced by its small companion (e.g., Orosz \& Hauschildt 2000). Such an analysis was carefully performed very recently to model the Kepler lightcurve of HAT-P-7 (Welsh et al. 2010). Instead, we are interested here only in order-of-magnitude approximation, so we can check the consistency of the detected amplitudes with the theory. All our approximations are evaluated for an inclination angle $i$ close to $90 \mathrm{deg}$, so we could ignore the $\sin i$ dependence of the three effects.

\subsection{Ellipsoidal effect}

To derive the order-of-magnitude of the ellipsoidal effect we used the analytical approximation of Morris \& Naftilan (1993), who used the Kopal (1959) expansion of the periodic variation into discrete Fourier series with terms that depend on the ratio $R_{*} / a$, where $R_{*}$ is the stellar radius and $a$ is the semi-major axis of the orbit (see also Pfahl et al. 2008). Assuming $R_{*} / a$ is small, the leading term of the stellar variation has a semi-amplitude of

$A_{\text {ellip }} \simeq \alpha_{\text {ellip }} \frac{m_{\mathrm{p}}}{M_{*}}\left(\frac{R_{*}}{a}\right)^{3}$,

where

$\alpha_{\text {ellip }}=0.15 \frac{(15+u)(1+g)}{3-u}$

is of order unity. In the above expression, $m_{\mathrm{p}}$ is the planetary mass, $M_{*}$ is the stellar mass, $g$ is the stellar gravity darkening coefficient, and $u$ is its limb-darkening coefficient (e.g., Mazeh 2008).

\subsection{Beaming effect}

For a circular orbit, the amplitude of the beaming effect can be written as

$A_{\text {beam }}=\alpha_{\text {beam }} 4 \frac{K_{r}}{c}$

where $K_{r}$ is the stellar radial-velocity amplitude and $c$ is the speed of light (Loeb \& Gaudi 2003; Zucker et al. 2007), and $\alpha_{\text {beam }}$ is of order unity. The factor $4 K_{r} / c$ represents the beaming effect for bolometric photometric observations, but ignores the Doppler shift photometric effect, which appears when the photometric observations are made in a specific bandpass, so that some of the stellar light is shifted out of or into the observed bandpass. The latter is accounted for by the $\alpha_{\text {beam }}$ factor, and we assume that for the CoRoT red bandpass it is of order unity.

\subsection{Reflection effect}

In our simplistic approximation we include in the reflection modulation the thermal emission from the dayside of CoRoT-3b, assuming both are modulated with the same phase (e.g., Snellen et al. 2009). The amplitude of the modulation of the reflected light alone is

$A_{\text {refl }}=p_{\text {geo }}\left(\frac{r_{\mathrm{p}}}{a}\right)^{2}$

where $r_{\mathrm{p}}$ is the planetary radius and $p_{\text {geo }}$ is the geometrical albedo (e.g., Rowe et al. 2008). Rowe et al. (2008) found quite a small albedo, of 0.03, for HD 209458, but recent study (Cowan \& Agol 2009) suggested that exoplanets may have a much larger albedo, of up to 0.5 . We therefore write the amplitude of the reflection effect, including the thermal emission, as

$A_{\text {refl }}=\alpha_{\text {refl }} 0.1\left(\frac{r_{\mathrm{p}}}{a}\right)^{2}$

where $\alpha_{\text {refl }}$ is of order unity. 
Table 2. CoRoT-3 parameters, as derived by Deleuil et al. (2008) and Triaud et al. (2009).

\begin{tabular}{lll}
\hline \hline Parameter & Derived value & Unit \\
\hline$a / R_{*}$ & $7.8 \pm 0.4$ & \\
$r_{\mathrm{p}} / R_{*}$ & $(663 \pm 9) \times 10^{-4}$ & \\
$m_{\mathrm{p}}$ & $21.7 \pm 1.0$ & $M_{\text {Jup }}$ \\
$M_{*}$ & $1.37 \pm 0.09$ & $M_{\odot}$ \\
$T_{*}$ & $6740 \pm 140 \mathrm{~K}$ & $\mathrm{deg}$ \\
$K_{r}$ & $2170 \pm 30$ & $\mathrm{~m} \mathrm{~s}^{-1}$ \\
\hline
\end{tabular}

\subsection{Application to CoRoT-3}

Table 2 presents the relevant parameters of CoRoT-3. The first four parameters were derived by Deleuil et al. (2008), while the last parameter, the radial-velocity amplitude, was deduced by Triaud et al. (2009). From these parameters we derived the expected values of the amplitudes of the ellipsoidal, beaming, and reflection effects, which are given in Table 1.

We emphasize that in each of the three theoretical amplitudes given in Table 1, the main source of uncertainty is hidden in the $\alpha$ factor, which we did not calculate. The numerical values, with their relatively small errors, are only order-of-magnitude approximations for inclination angles close to $90 \mathrm{deg}$.

The amplitudes derived from the cleaned lightcurve of CoRoT-3, as shown in Table 1, are of the same order of magnitude as the expected values, based on our simplistic approximation. This is true in particular for the beaming effect, where the theoretical approximation was found to be quite accurate. We therefore propose that we have detected the ellipsoidal and beaming effects of CoRoT-3. Our results suggest that the $\alpha_{\text {ellip }}$ factor in CoRoT-3 is on the order of 2. The reflection effect was too small to ensure a significant detection, given the SNR of the lightcurve.

\section{Discussion}

Our analysis has demonstrated that the red-channel lightcurve of CoRoT-3 includes the ellipsoidal and beaming effects. This is the first time that the beaming effect has been detected for substellar companion. We have been able to detect the two effects, with 59 and 27 ppm amplitudes, respectively, because of a combination of three features:

- CoRoT-3 brightness: with $r^{\prime}$-mag of 13.1, the star is among the brightest CoRoT targets, which are typically in the range of 11 to 16 in $r^{\prime}$ (Deleuil et al. 2008);

- the long observational run: the LRc01 lasted for 152 days, and the CCDs did not show yet any aging signals;

- the mass of CoRoT-3b: This massive-planet/brown-dwarf companion has one of the largest masses, $22 M_{\text {Jup }}$, discovered by CoRoT for substellar objects.

The last feature suggests that the stellar rotation has achieved synchronization with the orbital period of 4.3 days, without which the analysis of the ellipsoidal effect could have been more complicated.

Had this analysis been performed immediately after the discovery of the transits of CoRoT-3, and in particular before the radial-velocity confirmation of the planetary nature of the transiting object, we could have estimated the mass of the unseen object from the observed amplitudes of the ellipsoidal and beaming effects, provided we had been able to accurately derive their expected values. Such analysis could, in principle, save costly radial-velocity observations, or at least reduce to a minimum the number of observed velocities needed to confirm the substellar mass of the transiting object.

Obviously, the analysis of lightcurves obtained by space missions is dramatically different from those obtained by ground-based photometry. For the latter, the appearance of the ellipsoidal modulation in the data of transit candidates was considered a sign that the transiting object was of stellar nature, as suggested by Sirko \& Paczyński (2003), and applied, for example, by Kane et al. (2008) and Pietrukowicz et al. (2010). This is so because of the relatively high threshold of detection of the ellipsoidal modulation in the ground-based photometry. On the other hand, the present work, and the study of Welsh et al. (2010), suggest that the detection of the ellipsoidal modulation with a small amplitude in the CoRoT and Kepler data may indicate that the transiting object is a massive-planet/brown-dwarf object.

The present analysis suggests that, in principle, the three effects, or at least two of them, can be detected in the CoRoT lightcurves for some massive-planet/brown-dwarf objects, even without any transits, as suggested by Loeb \& Gaudi (2003) and Zucker et al. (2007). The effects can be stronger for systems with shorter orbital periods, and therefore can be detected in stars fainter than CoRoT-3 in the CoRoT fields. Many objects similar to CoRoT-3 should also be detected by Kepler, because of both higher SNR and longer timespan of its lightcurves.

Acknowledgements. We thank Shay Zucker for helpful discussions, Antonio Claret and Edward Guinan for help with the theoretical estimation of the ellipsoidal modulation, and Avi Shporer for careful reading of the paper and helpful suggestions. This research was supported by the Israel Science Foundation (grant No. 655/07).

\section{References}

Ahmed, N. T., Natarajan, T. K. R., \& Rao, K. R. 1974, IEEE Trans. Computers, 90

Auvergne, M., Bodin, P., Boisnard, L., et al. 2009, A\&A, 506, 411

Baglin, A., Auvergne, M., Boisnard, L., et al. 2006, 36th COSPAR Scientific Assembly, 36, 3749

Baudin, F., Baglin, A., Orcesi, J.-L., et al. 2006, ESA Spec. Pub., 1306, 145

Borucki, W. J., Koch, D., Basri, G., et al. 2010, Science, 327, 977

Cabrera, J., Fridlund, M., Ollivier, M., et al. 2009, A\&A, 506, 501

Cowan, N. B., \& Agol, E. 2009, ApJ, submitted [arXiv: 1001.0012]

Deleuil, M., Deeg, H. J., Alonso, R., et al. 2008, A\&A, 491, 889

Drake, A. J. 2003, ApJ, 589, 1020

Etzel, P. B. 1980, EBOP User's Guide, 3rd edn., UCLA A\&A

For, B.-Q., Green, E. M., Fontaine, G., et al. 2010, ApJ, 708, 253

Kane, S. R., Clarkson, W. I., West, R. G., et al. 2008, MNRAS, 384, 1097

Koch, D. G., Borucki, W. J., Basri, G., et al. 2010, ApJ, 713, L79

Kopal, Z. 1959, Close binary systems, The International Astrophysics Series (London: Chapman \& Hall)

Loeb, A., \& Gaudi, B. S. 2003,ApJ, 588, L117

Maxted, P. F. L., Marsh, T. R., \& North, R. C. 2000, MNRAS, 317, L41

Mazeh, T. 2008, EAS Pub. Ser., 29, 1

Mazeh, T., Guterman, P., Aigrain, S., et al. 2009, A\&A, 506, 431

Morris, S. L., \& Naftilan, S. A. 1993, ApJ, 419, 344

Orosz, J. A., \& Hauschildt, P. H. 2000, A\&A, 364, 265

Pál, A., Bakos, G. Á., Torres, G., et al. 2008, ApJ, 680, 1450

Pfahl, E., Arras, P., \& Paxton, B. 2008, ApJ, 679, 783

Pietrukowicz, P., Minniti, D., Díaz, R. F., et al. 2010, A\&A, 509, A4

Popper, D. M., \& Etzel, P. B. 1981, AJ, 86, 102

Rouan, D., Baglin, A., Copet, E., et al. 1998, Earth Moon and Planets, 81, 79

Rowe, J. F., Matthews, J. M., Seager, S., et al. 2008, ApJ, 689, 1345

Sirko, E., \& Paczyński, B. 2003, ApJ, 592, 1217

Snellen, I. A. G., de Mooij, E. J. W., \& Albrecht, S. 2009, Nature, 459, 543

Tamuz, O., Mazeh, T., \& North, P. 2006, MNRAS, 367, 1521

Triaud, A. H. M. J., Queloz, D., Bouchy, F., et al. 2009, A\&A, 506, 377

van Kerkwijk, M. H., Rappaport, S. A., Breton, R. P., et al. 2010, ApJ, 715, 51

Welsh, W. F., Orosz, J. A., Seager, S., et al. 2010, ApJ, 713, L145

Wilson, R. E., \& Devinney, E. J. 1971, ApJ, 166, 605

Zucker, S., Mazeh, T., \& Alexander, T. 2007, ApJ, 670, 1326 